\title{
Polynomial Growth for Birational Mappings from Four-State Spin Edge Models
}

\author{
$J-M$ MAILLARD \\ LPTHE, Université de Paris VI, Tour 24, 5ème étage, case 7109, 2 Place Jussieu, 75251 \\ Paris Cedex, France \\ E-mail: maillard@lpthe.jussieu.fr
}

This paper is part of the Proceedings of SIDE V;

Giens, June 21-26, 2002

\begin{abstract}
We classify all four-state spin edge models according to their behavior under a specific group of birational symmetry transformations generated from the so-called inversion relations. This analysis uses the measure of complexity of the action of birational symmetries of these lattice models, and aims at uncovering (star-triangle) solvable ones. One finds that these spin edge models have birational symmetries with a polynomial growth of the iteration calculations. We obtain an unexpected elliptic parametrization of the four-state chiral Potts model, as well as simple, and well-defined, examples of "transcendental" integrability compatible with this polynomial growth of the iteration calculations. As a byproduct we also obtain several homogeneous polynomial representation of the relative integers $\mathbf{Z}$ together with their multiplication.
\end{abstract}

\section{Introduction}

The results presented here originate in a long-standing collaboration of J-C Anglès d'Auriac, C-M. Viallet and the author [1]. In previous papers birational mappings [2, 3, 4] generated by involutive transformations on matrices have been studied. They have their origin in the theory of exactly solvable models in lattice statistical mechanics $[6,7,8,10,11,12]$. These birational mappings generically form an infinite discrete set of symmetries of lattice models. When the lattice model is Yang-Baxter integrable, that is star-triangle integrable for spin edge models, these birational mappings yield an infinite discrete set of symmetries of the Yang-Baxter, or star-triangle, equations [6, 7, 8, 10, 11, 12]. However, it is crucial to keep in mind that these birational transformations are infinite discrete set of symmetries of the lattice models even if the models are not Yang-Baxter integrable: in that generic (non Yang-Baxter integrable) case they are, for instance, symmetries of the phase diagram of the model [13]. These birational discrete symmetries of lattice models are generated by the inversion relations [14] which correspond, for spin edge lattice models, to combine a matrix inversion of a Boltzmann weight matrix together with the inversion of the entries of this matrix $[6,7,8,10,11,12]$. The iteration of the associated birational transformations presents some remarkable factorization properties $[3,4,5]$. These factorization 
properties explain why the degree of the successive rational expressions, one obtains when iterating the birational mappings, instead of having the exponential growth one expects at first sight, may have a polynomial growth of the complexity of these iterations $[3,4,9]$. As far as Yang-Baxter integrability is concerned, it has been shown [13, 14] that the Yang-Baxter, or star-triangle, equations are necessarily parametrized in terms of algebraic varieties having these birational transformations as automorphisms. Roughly speaking two situations are thus compatible with the Yang-Baxter, or star-triangle, integrability: either these birational transformations are infinite order, or these birational transformations are finite order and, writing down this finite order condition, enables to actually obtain the equations of these algebraic varieties ${ }^{1}$. The generic exponential growth of the calculations excludes the Yang-Baxter, or star-triangle, integrability. As far as growth of the iteration calculations are concerned, finite order birational transformations means that the degree of the successive rational expressions for the iterates is bounded (constant or periodic). The Yang-Baxter, or star-triangle, integrability thus correspond to growth of the iteration calculations which are at most polynomial (polynomial, constant or periodic). Beyond Yang-Baxter integrability the analysis of the symmetries of parameter space of lattice models imposes to analyze the same canonical, and natural, discrete dynamical systems corresponding to birational mappings generated from two involutions $[13,19]$. One can thus analyze these discrete dynamical systems as precious powerful tools for analysis lattice models, or, simply, for themselves.

\section{Spin edge $q$-state models.}

The models are defined on a $d$-dimensional regular lattice, each vertex bearing a spin $\sigma$ which can take $q$ values. One arranges the edge Boltzmann weight $W_{\sigma, \sigma^{\prime}}$ corresponding to the configurations of two nearest neighbor spin $\sigma$ and $\sigma^{\prime}$, in a $q \times q$ matrix $W$. A model is defined by giving the matrix of Boltzmann weights of local edge configurations: the state of an edge is determined by the values of the spin at its ends. One arranges the weights in a matrix $W$ whose entries $W_{i, j}$ are the weights of the edge configuration with one end in state $i$ and the other end in state $j$. One distinguishes different types of bonds, like for example for two-dimensional square lattices, the vertical and horizontal bonds, or, for most general non isotropic models, it is then associated to a number of $q \times q$ matrices $W_{\nu}$, one for each type of bond (checkerboard lattice ...). The entries of the matrices $W$ are, of course, defined up to an overall multiplicative factor. The most general matrix is then a collection of $q^{2}$ entries, defined up to a factor. Denoting $W$ and $\bar{W}$ the horizontal and vertical Boltzmann weights of an anisotropic square lattice, the inversion relation [14] amounts to performing, on the horizontal Boltzmann weight, the matrix inversion and, on the vertical Boltzmann weight, the (Hadamard) inverse $J$ :

$$
\begin{array}{ll}
I: & W_{k l} \longrightarrow\left(W^{-1}\right)_{k l}=A_{k l} / \operatorname{det}(W) \\
J: & W_{k l} \longrightarrow 1 / W_{k l}
\end{array}
$$

where $A_{k l}$ is the cofactor ${ }^{2}$ of $W_{k l}$ in $W$. The symmetry group is generated by simple involutive generators, namely the matrix inverse $I$, and the element by element inverse

\footnotetext{
${ }^{1}$ See for instance the higher genus integrable chiral Potts model $[15,16,20]$.

${ }^{2}$ Since the Boltzmann weights are defined up to overall multiplicative factors, one can, alternatively, also introduce a homogeneous inverse $I_{h}: \quad W_{k l} \longrightarrow A_{k l}=\operatorname{det}(W) \cdot\left(W^{-1}\right)_{k l}$
} 
$J$, both taken up to a overall multiplicative factor. The group generated by these two involutions is isomorphic to the infinite dihedral group $\mathbf{Z}_{2} \propto \mathbf{Z}$ since the most general element of this group of (birational) transformations is of the form $I^{\alpha} \cdot(I J)^{n}$ where $\alpha=$ 0,1 and $n$ is a relative integer (the inverse of $I J$ is $J I$ ). Up to the semi-direct product by a simple involution, the group of symmetry basically corresponds to the iteration (forward or backward) of one birational transformation : $K=I \cdot J$.

From now on, let us restrict to four-state spin edge models, that is $4 \times 4$ matrices. Considering the action of the birational transformation $K=I \cdot J$ on the most general $4 \times 4$ matrix, which means that one studies a birational transformation in $C P_{15}$ (sixteen homogeneous parameters), one will see, surprisingly, that one gets a polynomial growth of the iteration calculations!

\subsection{Spin edge models as specific patterns of a $4 \times 4$ matrix.}

In general, and for obvious simplicity reasons, one prefers, in lattice statistical mechanics, to consider models depending on small set of variables (two, three ...). This amounts to imposing symmetries on the model, namely, most of the time, equalities between various entries of the edge Boltzmann weight matrix, which are compatible with the general symmetries of the model (star-triangle integrability if any, inversion relations, gauge symmetries : see (2.8) below ...). For instance, let us consider the following four matrices ${ }^{3}$ :

$$
\begin{gathered}
M_{16}=\left[\begin{array}{llll}
w_{0} & w_{1} & w_{2} & w_{3} \\
w_{1} & w_{0} & w_{3} & w_{2} \\
w_{2} & w_{3} & w_{0} & w_{1} \\
w_{3} & w_{2} & w_{1} & w_{0}
\end{array}\right], \quad M_{17}=\left[\begin{array}{llll}
w_{0} & w_{1} & w_{2} & w_{3} \\
w_{3} & w_{0} & w_{1} & w_{2} \\
w_{2} & w_{3} & w_{0} & w_{1} \\
w_{1} & w_{2} & w_{3} & w_{0}
\end{array}\right], \\
M_{8}=\left[\begin{array}{llll}
w_{0} & w_{1} & w_{1} & w_{1} \\
w_{1} & w_{2} & w_{3} & w_{3} \\
w_{1} & w_{3} & w_{2} & w_{3} \\
w_{1} & w_{3} & w_{3} & w_{2}
\end{array}\right], \quad M_{36}=\left[\begin{array}{llll}
w_{0} & w_{1} & w_{2} & w_{2} \\
w_{3} & w_{0} & w_{4} & w_{4} \\
w_{4} & w_{2} & w_{5} & w_{6} \\
w_{4} & w_{2} & w_{6} & w_{5}
\end{array}\right]
\end{gathered}
$$

The first two matrices correspond respectively to the Ashkin-Teller model $[6,17]$ and the chiral Potts model $[15,16,20]$. One verifies immediately that the corresponding two matrices $M_{16}$ and $M_{17}$ are compatible with the involution $I$ (and of course involution $J)$ : their matrix inverse $M_{16}^{-1}$ and $M_{17}^{-1}$ are of the same form. One can verify that the matrix $M_{36}$, which depends on seven homogeneous parameters, is compatible with the involution $I$ (and of course $J$ ). Coming back to $M_{17}$, this matrix is obviously compatible with the matrix inversion $I$ : the inverse of a cyclic matrix is also a cyclic matrix. It is less obvious, at first sight, that the inverse of a matrix $M_{36}$ is of the same form as $M_{36}$. Let us one first note that the family of matrices $M_{36}$ can be simultaneously block-diagonalized,

\footnotetext{
${ }^{3}$ We use the indexation of matrices introduced in [1].
} 
$M_{36} \rightarrow \tilde{M}_{36}=R \cdot M_{36} \cdot R^{-1}:$

$$
\begin{aligned}
& \tilde{M}_{36}=\left[\begin{array}{cccc}
A & B & C & 0 \\
-B & D & E & 0 \\
C & -E & F & 0 \\
0 & 0 & 0 & G
\end{array}\right] \quad \text { with }: \quad R=\left[\begin{array}{cccc}
1 & 1 & 0 & 0 \\
1 & -1 & 0 & 0 \\
0 & 0 & 1 & 1 \\
0 & 0 & 1 & -1
\end{array}\right] \\
& A=2 w_{0}+w_{1}+w_{3}, \quad B=w_{3}-w_{1}, \quad C=2\left(w_{2}+w_{4}\right), \quad E=2\left(w_{2}-w_{4}\right), \\
& D=2 w_{0}-w_{3}-w_{1}, \quad F=2\left(w_{5}+w_{6}\right), \quad G=2\left(w_{5}-w_{6}\right)
\end{aligned}
$$

This block diagonalization, then, makes clear that the pattern $M_{36}$ is stable by the matrix inversion, since one can verify that the $3 \times 3$ upper block $B_{36}$ in (2.5) is a form of $3 \times 3$ block matrix actually stable by the matrix inversion. However, this form, $B_{36}$, is not stable by the (non-commutative) matrix product, but by the matrix square $\left(B_{36} \rightarrow B_{36}^{2}\right)$ :

$$
\begin{aligned}
& {\left[\begin{array}{ccc}
A^{\prime} & B^{\prime} & C^{\prime} \\
-B^{\prime} & D^{\prime} & E^{\prime} \\
C^{\prime} & -E^{\prime} & F^{\prime}
\end{array}\right]=\left[\begin{array}{ccc}
A & B & C \\
-B & D & E \\
C & -E & F
\end{array}\right] \cdot\left[\begin{array}{ccc}
A & B & C \\
-B & D & E \\
C & -E & F
\end{array}\right]} \\
& A^{\prime}=A^{2}-B^{2}+C^{2}, \quad B^{\prime}=A B+B D-C E, \\
& C^{\prime}=A C+B E+C F, \quad D^{\prime}=-B^{2}+D^{2}-E^{2}, \\
& E^{\prime}=-B C+D E+E F, \quad F^{\prime}=C^{2}-E^{2}+F^{2}
\end{aligned}
$$

The following three algebraic expressions are invariant under transformation (2.5) :

$$
\begin{aligned}
& \frac{-A B E+B E F+C E^{2}-B^{2} C}{B E^{2}+A C E-C D E+B C^{2}}, \quad \frac{B^{2} E+C^{2} E+B C F-B C D}{B E^{2}+A C E-C D E+B C^{2}}, \\
& \frac{C E^{2}-A B E+B D E+C^{3}+C D F-C D^{2}+A C D-A C F}{B E^{2}+A C E-C D E+B C^{2}}
\end{aligned}
$$

In terms of the eigenvalues $\lambda_{\alpha}$ of this $3 \times 3$ block matrix $B_{36}$, the transformation reads $\lambda_{\alpha} \rightarrow \lambda_{\alpha}^{2}$. Similarly, the n-th (matrix) power $B_{36}^{n}$ is also of the same form as the $3 \times 3$ block $B_{36}$, for any positive, or negative, integer $n$. One has, obviously, the same result on the original $4 \times 4$ pattern $M_{36}: M_{36}^{n}$ is also of the same form as $M_{36}$ for any positive or negative integer $n$ (and, of course, pattern $M_{36}$ is compatible with the Hadamard inverse $J$ ). The $M_{36} \rightarrow M_{36}^{2}$ transformation yields the following homogeneous quadratic transformation :

$$
\begin{array}{ll}
w_{0}^{\prime}=w_{0}^{2}+w_{1} w_{3}+2 w_{2} w_{4}, & w_{1}^{\prime}=2\left(w_{0} w_{1}+w_{2}^{2}\right), \\
w_{2}^{\prime}=w_{2} w_{0}+w_{1} w_{4}+w_{2} w_{5}+w_{2} w_{6}, & w_{3}^{\prime}=2\left(w_{0} w_{3}+w_{4}{ }^{2}\right), \\
w_{4}^{\prime}=w_{3} w_{2}+w_{0} w_{4}+w_{4} w_{5}+w_{4} w_{6}, & w_{5}^{\prime}=2 w_{2} w_{4}+w_{5}{ }^{2}+w_{6}{ }^{2}, \\
w_{6}^{\prime}=2\left(w_{2} w_{4}+w_{5} w_{6}\right) &
\end{array}
$$


while the $M_{36} \rightarrow M_{36}^{3}$ transformation yields the homogeneous cubic mapping :

$$
\begin{gathered}
w_{0}^{\prime \prime}=3 w_{1} w_{3} w_{0}+w_{0}^{3}+2\left(w_{1} w_{4}^{2}+w_{3} w_{2}^{2}\right)+2 w_{2} w_{4}\left(w_{5}+w_{6}+2 w_{0}\right) \\
w_{1}^{\prime \prime}=3 w_{1} w_{0}^{2}+w_{1}^{2} w_{3}+4\left(w_{1} w_{2} w_{4}+w_{0} w_{2}^{2}\right)+2\left(w_{2}^{2} w_{5}+w_{2}^{2} w_{6}\right) \\
w_{2}^{\prime \prime}=w_{1} w_{3} w_{2}+2\left(w_{1} w_{0} w_{4}+w_{2} w_{5} w_{6}\right)+w_{1} w_{4} w_{5}+w_{1} w_{4} w_{6} \\
\quad+w_{0}^{2} w_{2}+w_{0} w_{2} w_{5}+w_{0} w_{2} w_{6}+4 w_{2}^{2} w_{4}+w_{2} w_{5}^{2}+w_{2} w_{6}^{2} \\
w_{3}^{\prime \prime}=3 w_{3} w_{0}^{2}+w_{1} w_{3}^{2}+4 w_{3} w_{2} w_{4}+2 w_{4}^{2}\left(w_{5}+w_{6}+2 w_{0}\right) \\
w_{4}^{\prime \prime}=w_{3} w_{2}\left(w_{5}+w_{6}+2 w_{0}\right)+w_{0} w_{4}\left(w_{5}+w_{6}+w_{0}\right)+w_{3} w_{1} w_{4} \\
\quad+4 w_{2} w_{4}^{2}+w_{4} w_{5}^{2}+w_{4} w_{6}^{2}+2 w_{4} w_{5} w_{6} \\
w_{5}^{\prime \prime}=w_{3} w_{2}^{2}+2 w_{2} w_{4}\left(w_{0}+2 w_{5}+2 w_{6}\right)+w_{1} w_{4}^{2}+w_{5}^{3}+3 w_{5} w_{6}^{2} \\
w_{6}^{\prime \prime}=w_{3} w_{2}^{2}+2 w_{2} w_{4}\left(w_{0}+2 w_{5}+2 w_{6}\right)+w_{1} w_{4}^{2}+3 w_{6} w_{5}^{2}+w_{6}^{3}
\end{gathered}
$$

One easily verifies that these two homogeneous polynomial transformations (2.6) and (2.7) commute and correspond to an exponential degree growth of the iteration like, respectively, $2^{n}$ and $3^{n}$. More generally, the homogeneous polynomial transformations corresponding to $M_{36} \rightarrow M_{36}^{n}$, where $n$ is a positive or negative integer, form a set of transformations that commute together, that is to say a (homogeneous polynomial) representation of the relative integers $\mathbf{Z}$ together with their multiplication: $(m, n) \rightarrow m \cdot n$ with $m, n \in \mathbf{Z}$. Another example of pattern stable by the matrix inversion, is matrix $M_{5}$ which depends on three homogeneous parameters [1]. Matrix $M_{5}$ can be reduced, in a similar way using a fixed matrix $R$, to $\tilde{M}_{5}=R \cdot M_{5} \cdot R^{-1}$ :

$$
M_{5}=\left[\begin{array}{cccc}
w_{0} & w_{1} & w_{2} & w_{0} \\
w_{1} & w_{0} & w_{0} & w_{2} \\
w_{2} & w_{0} & w_{0} & w_{1} \\
w_{0} & w_{2} & w_{1} & w_{0}
\end{array}\right] \quad \longrightarrow \quad \tilde{M}_{5}=\left[\begin{array}{cccc}
A & 0 & 0 & 0 \\
0 & B & 0 & 0 \\
0 & 0 & -B & 0 \\
0 & 0 & 0 & C
\end{array}\right]
$$

Two matrices of the form $M_{5}$ obviously commute but their matrix product is clearly not of the same form, however the product of an odd number of such matrices is of the same form. Similarly matrix $M_{8}$ (see (2.4)) can be block-diagonalized by a fixed matrix into a $2 \times 2$ symmetric block and two equal $1 \times 1$ blocks (two identical eigenvalues). The matrix product of $n$ times the same matrix $M_{8}$ is of the same form. With these examples one sees that these set of matrices do not correspond to any obvious well-known structure (family of commuting matrices, group structure, ...). More generally, in the framework of fourstate spin edge models, seeking for an interesting model for lattice statistical mechanics, amounts to imposing equalities between the entries of a $4 \times 4$ matrix (thus reducing the $q^{2}=4^{2}$ homogeneous entries to $r$ homogeneous entries) such that these equalities between entries are also verified for the inverse matrix. We will call admissible pattern such a Icompatible pattern for a $4 \times 4$ matrix $^{4}$. These admissible patterns have been classified in [1] taking into account some symmetries that naturally pop out in this classification analysis.

Gauge equivalence and beyond. It is clear that there exist some "gauge-like" symmetries on the $q \times q$ matrix Boltzmann weight $W$. For instance relabeling the $q$ colors

\footnotetext{
${ }^{4}$ Equalities between entries are automatically $J$-compatible, so these admissible pattern are $K$ compatible, with $K=I \cdot J$.
} 
of the spin, obviously does not modify the partition function (transformation on dummy variables ...). In terms of the Boltzmann weight matrix $W$, this relabeling corresponds to a similarity transformation by a permutation matrix $P$ (see [1] for more details). As far as birational mappings are concerned, this also corresponds to a relabeling of the variables. One can also perform the following "gauge" transformations on the entries of the $4 \times 4$ Boltzmann weight matrix :

$$
W_{\sigma, \sigma^{\prime}} \quad \longrightarrow \quad W_{\sigma, \sigma^{\prime}}^{\prime}=W_{\sigma, \sigma^{\prime}} \cdot \frac{F(\sigma)}{F\left(\sigma^{\prime}\right)}
$$

The full "gauge" action can thus be represented by a similarity transformation $W \longrightarrow$ $W^{\prime}=g^{-1} \cdot W \cdot g$ where matrix $g=P \cdot D$ is the product of a permutation matrix $P$ and of a diagonal matrix $D$. More generally, let us consider the transformation

$$
T_{g}: \quad W \longrightarrow W^{\prime}=g_{1}^{-1} \cdot W \cdot g_{2} \quad \text { with } \quad g_{1}=P_{1} \cdot D_{1}, \quad g_{2}=P_{2} \cdot D_{2}
$$

Let us consider the square of transformation $K$, namely $K^{2}=I \cdot J \cdot I \cdot J$, one verifies immediately that $T_{g}$ and $K^{2}$ commute.

\section{Miscellaneous admissible patterns}

An exhaustive classification of all these admissible patterns has been obtained for $4 \times 4$ matrices and is given in [1]. Let us now give miscellaneous examples of these admissible patterns [1]. We are using the same indexation as the one introduced in [1]. It is worth noticing that there exists no admissible pattern depending on $r$ homogeneous parameters with $10<r<16(r=10$ and $r=16$ correspond respectively to the symmetric $4 \times 4$ matrix and to the most general $4 \times 4$ matrix).

One finds that the iteration of the birational transformation $K=I \cdot J$, associated with the most general $4 \times 4$ matrix (sixteen homogeneous parameters), yields a polynomial growth of the iteration calculations [1]. The various miscellaneous examples we sketch below, therefore, also yield polynomial growth of the iteration calculations. We give various miscellaneous examples in order to show that a birational mapping with polynomial growth of the iteration calculation can actually correspond to various kind of orbits for the iteration of $K$ : rational or elliptic curves, abelian surfaces [5, 13], transcendental curves restricted to some abelian variety ...

\subsection{Pattern \#7: Symmetric Ashkin-Teller model}

Let us consider a subcase of the Ashkin-Teller model $M_{16}$ (see (2.3)), namely the symmetric Ashkin-Teller model [17], which corresponds to impose $w_{3}=w_{2}$ in $M_{16}$, thus defining a matrix pattern $M_{7}$. This matrix $M_{7}$ cannot be reduced to matrix $M_{5}$ by a simple relabeling (similarity transformation), however these two matrices are equivalent up to independent row and column relabeling (2.9) and, thus, correspond to the same birational transformation $K^{2}$. There is one algebraic $K$-invariant

$$
\Delta_{7}=\frac{w_{0} w_{1}-w_{2}^{2}}{w_{2}\left(w_{1}-w_{0}\right)}
$$

The curves $\Delta_{7}=a$ have a simple well-known rational parametrization: $w_{0}=t, \quad w_{1}=$ $(1-a t) /(t-a), \quad w_{2}=1$. 


\subsection{Pattern \#8 : transcendental integrability}

Let us consider the Boltzmann matrix $M_{8}$ given above in (2.3).

$$
\Delta_{8}^{(1)}=\frac{w_{2} w_{1}^{2}+2 w_{1}^{2} w_{3}-2 w_{0} w_{3} w_{2}-w_{0} w_{3}^{2}}{w_{2} w_{1}^{2}-w_{0} w_{3}^{2}}
$$

is invariant by $J$ and changes sign under the action of $I$. The action of $K$ exchanges the surfaces $\Delta_{8}^{(1)}=a$ and $\Delta_{8}^{(1)}=-a$. Since the condition $\Delta_{8}^{(1)}=a$ may be solved rationally in $w_{0}$, one may write the action of $K^{2}$ restricted to $\Delta_{8}^{(1)}=a$, with the coordinates $w_{1}, w_{2}, w_{3}$. In terms of the inhomogeneous variables $x=w_{1} / w_{3}$ and $y=w_{3} /\left(w_{2}+w_{3}\right)$, it reads:

$$
\begin{aligned}
& x \longrightarrow x^{\prime}=x \cdot \frac{(b+b y-y)}{(2+y)(b y-2 y+b-1)} \\
& y \longrightarrow y^{\prime}=y+b
\end{aligned}
$$

with $b=2 a /(1+a)$, and the quantity

$$
\begin{aligned}
\Delta_{8}^{(2)} & =x \cdot \Phi(y) \quad \text { with } \\
\Phi(y) & =\Gamma\left(\frac{y-2+b}{b}\right) \Gamma\left(\frac{y+1}{b}\right) / \Gamma\left(\frac{y-1+b}{b}\right) / \Gamma\left(\frac{y}{b}\right)
\end{aligned}
$$

can be seen to be invariant by the action of $K^{2}$ on $\Delta_{8}^{(1)}=a$. This example possesses a "mixture" of algebraic and non-algebraic invariants, which can be evaluated exactly. The orbits of $K$ are confined to non-algebraic curves, and the n-th iterate may be written explicitly :

$$
y_{n}=y_{0}+n b, \quad x_{n}=x_{0} \frac{\Phi\left(y_{0}\right)}{\Phi\left(y_{n}\right)}
$$

The orbits accumulate to the point $\left(x_{\infty}, y_{\infty}\right)=\left(\Delta_{8}^{(2)}\left(x_{0}, y_{0}\right), \infty\right)$, which is a point on the line $w_{2}+w_{3}=0$.

\subsection{Pattern \#17: Chiral Potts model}

The chiral Potts model [15, 16] corresponds to pattern \#17 in [1]. Its Boltzmann matrix was already given above (see $M_{17}$ in $(2.3)$ ). There exist two algebraically independent $K$-invariants :

$$
\Delta_{\text {Potts }}^{(1)}=\frac{w_{3} w_{2}-w_{0} w_{1}}{w_{1} w_{2}-w_{0} w_{3}}, \quad \Delta_{\text {Potts }}^{(2)}=\frac{\left(w_{0} w_{2}-w_{1}^{2}\right)\left(w_{0} w_{2}-w_{3}^{2}\right)}{\left(w_{0} w_{3}-w_{1} w_{2}\right)^{2}}
$$

The condition $\Delta_{\text {Potts }}^{(1)}=a$ can be solved rationally in $w_{0}$. Setting $\Delta_{\text {Potts }}^{(2)}=b$, together with $x=w_{1} / w_{2}$ and $y=w_{3} / w_{2}$, yields the equation of an elliptic curve to which the orbit is confined :

$$
(x-y)^{2}(x+y)^{2} b-\left(a x-y-a y x^{2}+x^{3}\right)\left(a x-y+y^{2} x-a y^{3}\right)=0
$$


One can actually see that the set of iterates of $K$ is dense in a curve. The transformations $I$ and $J$ may be restricted to the quadrics $\Delta_{\text {Potts }}^{(1)}=a=c s t$. They may be written on $x$ and $y$, for fixed $a$ :

$$
\begin{aligned}
i_{a}: & x \longrightarrow x^{\prime}=\frac{2 a x y^{2}-2 a y-a^{2} x^{2} y+a^{2} x+x-y^{3}}{1-a y^{2}+a^{2} x y-x y-a^{2}+a x^{2}} \\
y & \longrightarrow y^{\prime}=\frac{x y^{2}-2 a x^{2} y-y-a^{2} y+2 a x+a^{2} x^{3}}{1-a y^{2}+a^{2} x y-x y-a^{2}+a x^{2}} \\
j_{a}: \quad x \longrightarrow x^{\prime} & =1 / x, \quad y \longrightarrow y^{\prime}=1 / y
\end{aligned}
$$

The two involutions $i_{a}$ and $j_{a}$ leave the curves (3.5) globally invariant whatever $a$ and $b$ are. The modular invariant of the elliptic curve (3.5) is given by

$$
\begin{aligned}
& \begin{aligned}
j= & 1728 \frac{g_{2}^{3}}{g_{2}^{3}-27 g_{3}^{2}}=256 \frac{\left(1-\mathcal{M}+\mathcal{M}^{2}\right)^{3}}{\mathcal{M}^{2}(1-\mathcal{M})^{2}}, \\
g_{2}= & 1+256 b^{4}-512 a b^{3}-16 b^{2} a^{4}+288 b^{2} a^{2}-16 b^{2}+16 a^{5} b \\
& -32 a^{3} b+16 a b-4 a^{6}+a^{8}-4 a^{2}+6 a^{4}
\end{aligned} \\
& \begin{aligned}
g_{3}= & \left(1-32 b^{2}+32 a b-2 a^{2}+a^{4}\right)\left(1+16 b^{2}-16 a b-2 a^{2}+a^{4}\right) \\
& \times\left(1-8 b^{2}+8 a b-2 a^{2}+a^{4}\right) /(3 \sqrt{3})
\end{aligned} \\
& \text { and }: \quad \mathcal{M}=\frac{\left(1-2 a+a^{2}+4 b\right)\left(1+2 a+a^{2}-4 b\right)}{(1-a)^{2}(1+a)^{2}}
\end{aligned}
$$

$\mathcal{M}$ is the square of the modulus of the elliptic functions which parametrize the curve. Notice that $\mathcal{M}$ can, equivalently, be replaced in the modular invariant $j$, by any of the six values $\{\mathcal{M}, 1-\mathcal{M}, 1 / \mathcal{M}, 1-1 / \mathcal{M}, 1 /(1-\mathcal{M}), \mathcal{M} /(\mathcal{M}-1)\}$. Instead of the canonical Weierstrass form (see (3.11) below) the elliptic curve (3.5) can also be reduced to a symmetric biquadratic form [13] where the action of $K$ on some spectral parameter $\theta$, is crystal clear:

$$
\begin{aligned}
& \left(J_{x}-J_{y}\right)\left(p^{2} q^{2}+1\right)-\left(J_{x}+J_{y}\right)\left(p^{2}+q^{2}\right)+4 J_{z} p q=0, \\
& \text { with : } \quad J_{x}=2 a, \quad J_{y}=4 b-2 a, \quad J_{z}=a^{2}+1 \quad \text { and } \\
& \mathcal{M}=\frac{J_{z}{ }^{2}-J_{y}{ }^{2}}{J_{z}{ }^{2}-J_{x}{ }^{2}}, \quad p=\operatorname{sn}(\theta), \quad q=\operatorname{sn}(\theta \pm \lambda)
\end{aligned}
$$

Changing $\mathcal{M}$ to $1-\mathcal{M}$, or $1 / \mathcal{M}$, amounts to permuting $J_{x}, J_{y}$, and $J_{z}$. For given values of $a$ and $b$, the action of $K$ is a shift on the elliptic curve (3.5). It is possible to write down the Weierstrass form of the elliptic curve

$$
X^{3}-\alpha X-\beta+Y^{2}=0
$$

$$
\text { with : } \quad \alpha=\frac{1}{48} \frac{g_{2}}{(b-a)^{8}}, \quad \beta=\frac{\sqrt{3}}{288} \frac{g_{3}}{(b-a)^{12}}
$$

and give an explicit coordinate transformation [1] from the original variables $(x, y)$ to $[X, Y]$. The formula can be obtained through van Hoeij algorithm, after setting $b=$ $a+1 / u^{2}$ (or $u= \pm 1 / \sqrt{b-a}$, see [1]). The elliptic curve (3.5), or the previous symmetric 
biquadratic form (3.9), depends on the modulus $\mathcal{M}$ and on the shift $\lambda$ in (3.10), or equivalently, $j$ and $\lambda$. The Weierstrass form (3.11) depends only on $j$, up to straightforward rescaling. One may thus think that (3.11) is "blind" to the shift $\lambda$ in (3.10), and thus to the action of $K$. In fact, the action of $K$, when written on the Weierstarss form (3.11) is the addition ${ }^{5}$ of a point $\Pi_{+}$with coordinates $\left[X_{\pi_{+}}, Y_{\pi_{+}}\right]$(see [1] for more details). The fact that (3.11) does not depend on $\lambda$ is in agreement with the fact that the change of variables $(x, y) \rightarrow[X, Y]$ actually depends on $u$. In other words one could say that (3.5) is equivalent to (3.11) together with a singled-out point corresponding to the shift, namely $\Pi_{+}$, or (3.12) below. The inverse map $K^{-1}$ is the addition of the opposite point $\Pi_{-}=\left[X_{\pi_{+}},-Y_{\pi_{+}}\right]$on (3.11). The sum of $\Pi_{ \pm}$with itself, denoted $2 \cdot \Pi_{ \pm}$and the multiples $n \cdot \Pi_{ \pm}$can be obtained by recurrence. Their $[X, Y]$ coordinates may be re-expressed rationally in terms of $a$ and $b$. The $(x, y)$ coordinates of point $2 \cdot \Pi_{+}$corresponds to :

$$
(x, y)=\left(\frac{\left(1+a^{2}\right) a u}{1-u^{2} a^{3}-a^{2}}, \frac{\left(2+a u^{2}\right) a^{2} u}{1-u^{2} a^{3}-a^{2}}\right)
$$

of the original elliptic curve (3.5). Generic orbits of $K=I . J$ are infinite, but the degenerate cases, where these orbits are finite, play an important role in lattice statistical mechanics : this is where higher genus Yang-Baxter integrability takes place [20]. The condition $K^{(m+n)}=I_{d}$ (where $I_{d}$ denotes the identity transformation) is just :

$$
X\left(n \cdot \Pi_{+}\right)=X\left(m \cdot \Pi_{-}\right) \quad \text { and } \quad Y\left(n \cdot \Pi_{+}\right)=-Y\left(m \cdot \Pi_{-}\right)
$$

As an example, the condition that $K$ is of order four is an algebraic condition on the entries of the Boltzmann matrix, which corresponds to the higher genus star-triangle integrability of the chiral Potts model [20]. It is the vanishing of $Y\left(2 \cdot \Pi_{+}\right)$i.e. $\left(1+a^{2}\right)(2 b-a) a=0$ and, more specifically, condition $2 b-a=0$ (see [13]).

\subsection{Pattern \#25 : another transcendental integrability}

This example is a good illustration of a birational mappings with polynomial growth together with non-algebraic curves. The Boltzmann matrix reads :

$$
W_{25}=\left[\begin{array}{llll}
w_{0} & w_{1} & w_{2} & w_{3} \\
w_{4} & w_{0} & w_{4} & w_{2} \\
w_{2} & w_{3} & w_{0} & w_{1} \\
w_{4} & w_{2} & w_{4} & w_{0}
\end{array}\right]
$$

The number of inhomogeneous parameters is four. Two independent algebraic $K$-invariants can be found :

$$
\Delta_{25}^{(1)}=\frac{w_{0} w_{1}-w_{3} w_{2}}{w_{0} w_{3}-w_{1} w_{2}}, \quad \Delta_{25}^{(2)}=\frac{\left(w_{4} w_{3}-w_{0}^{2}\right)\left(w_{1} w_{4}-w_{2}^{2}\right)}{\left(w_{4} w_{3}-w_{2}^{2}\right)\left(w_{1} w_{4}-w_{0}^{2}\right)}
$$

The equation of the quadric $\Delta_{25}^{(1)}=a$, can be solved for $w_{1}$. One gets a representation of $I$ and $J$ restricted to $\Delta_{25}^{(1)}=a$. Changing the $w_{i}$ 's variables to $[x, y, z, t]=$

\footnotetext{
${ }^{5}$ To add two points on the cubic (3.11), draw the straight line through the two points. Compute the third point of intersection of the line with the curve, and take its symmetric under $Y \rightarrow-Y$.
} 
$\left[w_{0}^{2}, w_{0} w_{2}, w_{0} w_{3}, w_{3} w_{4}\right]$, one obtains :

$$
\begin{aligned}
i_{a}([x, y, z, t])=\quad & {\left[x(x+a y-a t-t)^{2}(x+y)(x+a y),\right.} \\
& (x+y)\left(a x t-x y+x t-a y^{2}\right)(x+a y)(x+a y-a t-t), \\
& -z(x+y)\left(x^{2}+x t a^{2}-x t-y^{2} a^{2}\right)(x+a y-a t-t), \\
& \left.(x+a y)(x-y) t\left(x^{2}+x t a^{2}-x t-y^{2} a^{2}\right)\right]
\end{aligned}
$$

the remaining algebraic invariant $\Delta_{25}^{(2)}$ becoming :

$$
\Delta_{25}^{a}=\frac{x(t-y)\left(y t-x y-a y^{2}+a x t\right)}{(x-y)^{2} t(x+y)}
$$

Notice that the third coordinate $z$, in equation (3.15), is just multiplied by a factor depending on the other coordinates, and does not appear anywhere else in the induced transformation $i_{a}$, nor in the invariant $\Delta_{25}^{a}$. The curves $\Delta_{25}^{a}=b$ are elliptic curves in the variables $(x, y, t)$, extending to cylinders in $[x, y, z, t]$. We may examine a particular value of $b$ where the curve $\Delta_{25}^{a}=b$ degenerates to a rational curve, but there exists a transcendent invariant for $i_{a}$. The rational parametrization of the cylinder is

$$
[x, y, z, t]=\left[\frac{s^{2} t(1+s a)}{s+a}, \frac{s t(1+s a)}{s+a}, z, t\right]
$$

The induced map $(I \cdot J)^{2}$ may be written

$$
\begin{aligned}
S \longrightarrow q^{2} S, & z \longrightarrow \frac{T(S)}{T(q S)} & \text { with } \quad T(S)=\frac{(1-S)\left(1-q^{3} S\right)}{\left(1-q^{2} S\right)\left(1-q^{5} S\right)} \\
\text { and } & S=\frac{s-q^{-1}}{s-q}, & q=-1 / 2 \frac{a+1+\sqrt{(1-a)(1+3 a)}}{a}
\end{aligned}
$$

Introducing an infinite product $\Pi(S)$ the $2 n$-th iterate of $K=I \cdot J$ reads :

$$
S_{2 n}=q^{4 n} S_{0}, \quad z_{2 n}=z_{0} \cdot \frac{\Pi\left(S_{0}\right)}{\Pi\left(S_{2 n}\right)} \quad \text { with } \quad \Pi(S)=\prod_{k=0}^{k=\infty} \frac{T\left(q^{4 k} S\right)}{T\left(q^{4 k+1} S\right)}
$$

In other words, the transcendent quantity $\Delta=z \cdot \Pi(S)$ is invariant by $K^{2}$. The convergence of these Eulerian products is ensured when $a \in(-1 / 3,1)$. Such values of $a$ would then produce orbits with accumulation points. If $a$ is outside this interval, then $q$ has unit modulus and one clearly sees curves for the orbits of $K$.

\section{Generalization to $q \times q$ matrices}

All these results can be performed for $q$-state edge Potts models, that is for $q \times q$ matrices instead of $4 \times 4$ matrices. This has been done for $q=3$ exhaustively, also yielding to a polynomial growth of the iteration calculations and also to several finite order situations [1]. For $q \geq 5$ the exploration of the admissible matrix patterns, stable by the matrix inversion, becomes a huge task $\left(\simeq 4.6 \cdot 10^{18}\right.$ patterns to explore $\left.\ldots\right)$. We have however performed a systematic analysis of the $5 \times 5$ matrices depending on three homogeneous parameters. It seems that one does not have pattern different from the above mentioned 
patterns (cyclic, symmetric matrix, most general). In particular one finds a "no-man's land" between the cyclic matrix and the symmetric matrix : there are no pattern depending on $r$ homogeneous parameters with $q<r<q(q+1) / 2(q=5)$, and probably a no-man's land, as well, between the symmetric matrix and the most general matrix. One could conjecture this could be the case for any prime number greater or equal to five.

We have studied, quite systematically, $6 \times 6$ patterns depending on a restricted number of parameters (namely three, four is already too much ...) such that all the diagonal terms are equal. We found that way a $6 \times 6$ matrix which is a stable pattern by $I$ (and $J$ of course) and corresponds to a six-state chiral Potts model in lattice statistical mechanics [19]. The birational transformation yields elliptic curves (linear pencil of elliptic curves) and thus corresponds to a polynomial growth of the calculations.

For arbitrary $q$ values there always exists some singled out patterns : the standard scalar Potts model pattern depending on two homogeneous parameters (all the diagonal terms are equal, all the off-diagonal terms are equal), the cyclic matrix depending on $q$ homogeneous parameters, the cyclic and symmetric matrix, the symmetric matrix depending on $q(q+1) / 2$ homogeneous parameters, and the most general matrix depending on $q^{2}$ homogeneous parameters (and of course the patterns one can deduce from independent relabeling of rows and columns). It seems that for $q=5$ one only gets these singled out cases and nothing else. It is clear that, when $q$ is not a prime number, one can get many more patterns. For instance, considering two $q \times q$ matrices of a $I$-compatible pattern, namely $A$ and $B$, the $2 q \times 2 q$ matrix (here $I_{d}$ is the $q \times q$ identity matrix):

$$
\left[\begin{array}{ll}
A & B \\
B & A
\end{array}\right]=\frac{1}{2} \cdot\left[\begin{array}{cc}
I_{d} & I_{d} \\
I_{d} & -I_{d}
\end{array}\right] \cdot\left[\begin{array}{cc}
A+B & 0 \\
0 & A-B
\end{array}\right] \cdot\left[\begin{array}{cc}
I_{d} & I_{d} \\
I_{d} & -I_{d}
\end{array}\right]
$$

is also a pattern stable by the matrix inversion $I$. The results described in section (2.1), and in particular the patterns $M_{36}$, the blocks $B_{36}$, and their stability property with respect to $M \rightarrow M^{n}$ where $n$ is any positive or negative integer (see (2.5)), can be generalized to $q \times q$ matrices for values of $q$ larger than 3 or 4 . For instance, one can easily guess a generalization of the blocks $B_{36}$ to $q \times q$ matrices and verify that these forms are stable not only by the matrix inversion, but also by $M \rightarrow M^{n}$. From this form one can deduce (for instance) $(q+1) \times(q+1)$ patterns stable by the matrix inversion and also by $M \rightarrow M^{n}$. Let us give this form for a $5 \times 5$ matrix and one (deduced) pattern, stable by the matrix inversion and also by $M \rightarrow M^{n}$, for a $6 \times 6$ matrix :

$$
\left[\begin{array}{ccccc}
a & b & c & h & k \\
-b & d & e & i & l \\
c & -e & f & j & m \\
-h & i & -j & g & n \\
k & -l & m & -n & o
\end{array}\right], \quad\left[\begin{array}{cccccc}
w_{0} & w_{1} & w_{2} & w_{3} & w_{4} & w_{4} \\
w_{5} & w_{0} & w_{6} & w_{7} & w_{8} & w_{8} \\
w_{7} & w_{3} & w_{9} & w_{10} & w_{11} & w_{11} \\
w_{6} & w_{2} & w_{12} & w_{9} & w_{13} & w_{13} \\
w_{8} & w_{4} & w_{13} & w_{11} & w_{14} & w_{15} \\
w_{8} & w_{4} & w_{13} & w_{11} & w_{15} & w_{14}
\end{array}\right]
$$

yielding homogeneous polynomial representation of $\zeta \rightarrow n \cdot \zeta$, with sixteen homogeneous parameters! Again, this provides (homogeneous polynomial) representations of the relative integers $\mathbf{Z}$ together with their multiplication: $(m, n) \rightarrow m \cdot n$. 


\section{Back to statistical mechanics}

All these results can be seen as interesting results on a certain set of rational transformations : integrable mappings yielding elliptic foliations, "transcendental" integrability, polynomial growth of the iteration calculations, ... Coming back to the lattice statistical mechanics origin of these birational transformations, one obtains many interesting results.

Star-triangle integrability. First, as far as star-triangle integrability is concerned, the existence of "transcendental" integrability rules out, generically, the star-triangle integrability. It may, however, be possible, when the transcendental invariants reduce to algebraic ones, that star-triangle integrability could be possible. This will thus occur on an algebraic subvariety of this pattern which rules out, generically, the star-triangle integrability.

Seeking for star-triangle integrability one can show that the star-triangle relation (if any ...) necessarily yields constraints on the matrix Boltzmann weights. Let us denote $W_{1}$, $W_{2}, W_{3}$ the three Boltzmann weights in the "star" and $\bar{W}_{1}, \bar{W}_{2}, \bar{W}_{3}$ the three Boltzmann weights in the "triangle". If one denotes the dyadic product ${ }^{6}$ of two Boltzmann weight matrix $W_{2}, W_{3}$ by $W_{23}=W_{2} \star W_{3}$, and by $\operatorname{Diag}\left(\bar{W}_{1}\right)$ the diagonal matrix built from the diagonal entries of matrix $\bar{W}_{1}$, and denoting by a dot the standard matrix product, one finds the following necessary conditions ${ }^{7}$ for the star-triangle relations :

$$
W_{1} \cdot\left(W_{2} \star W_{3}\right)=\left(\bar{W}_{2} \star \bar{W}_{3}\right) \cdot \operatorname{Diag}\left(\bar{W}_{1}\right)
$$

In order to verify this relation one must explore the following situations : either matrix $\operatorname{Diag}\left(\bar{W}_{1}\right)$ is proportional to the identity matrix and, thus, the necessary conditions (5.1) become :

$$
W_{1} \cdot\left(W_{2} \star W_{3}\right)=W_{1} \cdot W_{23}=\lambda \cdot\left(\bar{W}_{2} \star \bar{W}_{3}\right)=\lambda \cdot \bar{W}_{23}
$$

since the dyadic product of two matrices in the same pattern is necessarily a matrix in the same pattern. Therefore the star-triangle patterns must be stable by the matrix product. Denoting by $V$ the vector with all its entries equal to 1 , another necessary condition for the star-triangle relation reads :

$$
\left(W_{1} \star W_{2} \star W_{3}\right) \cdot V=\operatorname{Diag}\left(\bar{W}_{2}\right) \star \operatorname{Diag}\left(\bar{W}_{3}\right) \cdot \operatorname{Diag}\left(\bar{W}_{1}\right)
$$

One has to keep track of the orientation of the matrices (chiral models) : sometimes in the necessary equations (5.2) and (5.3), the $W_{i}$ or $\bar{W}_{i}$ can be changed into their transpose: $W_{i}^{t}$ or $\bar{W}_{i}^{t}$. If $W_{1} \star W_{2} \star W_{3}$ is another matrix in the pattern and if $\operatorname{Diag}\left(\bar{W}_{i}\right)$ is proportional to the identity, the previous relation (5.3) means that the pattern of matrices is a pattern of stochastic matrices. Let us denote $D_{1}$ the diagonal matrix such that $D_{1} \cdot V=W_{1} \cdot V$, another necessary condition for the star-triangle relation reads :

$$
W_{2} \cdot D_{1} \cdot W_{3}=\left(\bar{W}_{3} \cdot \bar{W}_{2}\right) \star \bar{W}_{1}
$$

Again this reinforces the "prejudice" that one should have stochastic, or bistochastic, matrices, and/or that the pattern of matrices is a pattern stable by the matrix product.

\footnotetext{
${ }^{6}$ The entries of the dyadic product $W_{23}=W_{2} \star W_{3}$ are the product of the entries of $W_{2}$ and $W_{3}$.

${ }^{7}$ This is just a quick sketch. In fact on has to take care of the orientation of the edges : $W$ may become the transpose $W^{t} \ldots$
} 
Therefore one has to study all the following possible scenario : 1) the $W_{i}$ or $\bar{W}_{i}$ are symmetric matrices or the dyadic product of a matrix in the pattern with a transpose of a matrix in the pattern $W \star\left(W^{\prime}\right)^{t}$, is also in the pattern (cyclic matrices for instance),

2 ) the $W_{i}$ 's (and $\bar{W}_{i}$ 's) form a group

$3)$ the $W_{i}$ 's and $\bar{W}_{i}$ 's are a commuting family of matrices

4) the $W_{i}$ or $\bar{W}_{i}$ are stochastic, or bi-stochastic, matrices. Actually the patterns corresponding to bi-stochastic, matrices are patterns number $1,2,3,4,5,6,7,10,11,16,17$ (with the notations of [1]) which correspond to models of lattice statistical mechanics for which star-triangle relations have been shown to exist.

As far as series analysis are concerned, one requires "transmissivity" variables in order to be able to perform series expansions. This can only exist for matrix Boltzmann weights reducing to diagonal matrices, that is commuting family of transfer matrices : this imposes that the pattern must depend at most on four homogeneous variables (pattern number \#8 is, for instance, ruled out).

\subsection{Conclusion}

The four-state edge spin models are seen to yield remarkable polynomial growth for the associated birational transformations. The various patterns that naturally pop out from the analysis of these birational transformations all provide interesting singled-out models for lattice statistical mechanics. Our results can be seen as discrete dynamical systems results or, coming back to statistical mechanics, as preliminary results when seeking for star-triangle integrability. Actually the transcendental integrability, we have found, generically rules out star-triangle integrability. However, even if the lattice models are not startriangle integrable, further analysis can be performed. One can seek for subvarieties in the parameter space such that the models becomes integrable. The finite order conditions for these birational mappings, $K^{n}=I_{d}$, are a "gold mine" for higher genus Yang-Baxter integrability and could be studied per se. One can also study the critical manifolds of these various (generically non-integrable) models. For instance, the location of the critical manifolds of these various models is a very interesting subject of analysis and our previous studies is precious in order to localize such manifolds, hopefully algebraic varieties.

Acknowledgments: I thank Euresco for financial support.

\section{References}

[1] J-C. Anglès d'Auriac, J-M. Maillard and C. M. Viallet, A classification of four-state spin edge Potts models. J. Phys. A 35 (2002) pp. 9251-9272 cond-mat/0209557

[2] S. Boukraa, J-M. Maillard and G. Rollet, Almost integrable mappings. Int. J. Mod. Phys. B8 (1994), 137-174.

[3] S. Boukraa J-M. Maillard and G. Rollet, Determinantal identities on integrable mappings. Int. J. Mod. Phys. B8 (1994), 2157-2201.

[4] S. Boukraa, J-M. Maillard and G. Rollet, Integrable mappings and polynomial growth. Physica A 209 (1994) 162-222 
[5] S. Boukraa et J-M. Maillard, Factorization properties of birational mappings. Physica A 220 (1995), 403-470.

[6] M.P. Bellon, J-M. Maillard and C-M. Viallet, Integrable Coxeter Groups. Phys. Lett. A 159 (1991), 221-232.

[7] M.P. Bellon, J-M. Maillard and C-M. Viallet, Higher dimensional mappings. Phys. Lett. A 159 (1991), 233-244.

[8] M.P. Bellon, J-M. Maillard and C-M. Viallet, Infinite Discrete Symmetry Group for the YangBaxter Equations: Spin models. Phys. Lett. A 157 (1991), 343-353.

[9] J. Diller and C. Favre, Dynamics of bimeromorphic maps of surfaces. Amer. J. Math. 123 (2001), no. 6, 1135-1169.

[10] M.P. Bellon, J-M. Maillard and C-M. Viallet, Infinite Discrete Symmetry Group for the YangBaxter Equations: Vertex Models. Phys. Lett. B 260 (1991), 87-100.

[11] M.P. Bellon, J-M. Maillard and C-M. Viallet, Rational Mappings, Arborescent Iterations, and the Symmetries of Integrability. Phys. Rev. Lett. 67 (1991), 1373-1376.

[12] M.P. Bellon, J-M. Maillard and C-M. Viallet, Quasi integrability of the sixteen-vertex model. Phys. Lett. B 281 (1992), 315-319.

[13] S. Boukraa, and J-M. Maillard, Let's Baxterise. J. Stat. Phys. Vol 102 (2001) 641-700 and : hep-th/0003212

[14] J-M. Maillard, Automorphisms of algebraic varieties and Yang-Baxter equations. J. Math. Phys. 27 (1986), 2776

[15] H. Au-Yang and B.M. Mc Coy and J.H.H. Perk and S. Tang and M.L. Yan", Commuting transfer matrices in the chiral Potts models: solutions of the star-triangle equations with genus Z 1. Phys. Lett. A 123, (1987) 219.

[16] R.J. Baxter and J.H.H. Perk and H. Au-Yang, New solutions of the star-triangle relations for the chiral Potts model. Phys. Lett. A 128, (1988) 138.

[17] J.M Maillard, P. Rujan, T. Truong, Algebraic properties and symmetries of the symmetric Ashkin-Teller model, J. Phys. A18 (1985) 339

[18] S. Boukraa, J-M. Maillard and G. Rollet, Discrete Symmetry Groups of Vertex Models in Statistical Mechanics. J. Stat.Phys. 78 (1995), 1195-1251.

[19] H.Meyer, J-C. Anglès d'Auriac, J-M. Maillard and G. Rollet, Phase diagram of a six-state chiral Potts model. Physica A 208 (1994), pp. 223-236.

[20] D. Hansel and J-M. Maillard, Symmetries of models with genus > 1. Phys. Lett. A 133, (1988), 11-15

[21] N. Abarenkova, J.-C. Anglès d'Auriac, S. Boukraa, S. Hassani and J.-M. Maillard, Rational dynamical zeta functions for birational transformations. Physica A 264 (1999), 264-293

[22] N. Abarenkova, J-C. Anglès d'Auriac, S. Boukraa, S. Hassani and J-M. Maillard, Topological entropy and complexity for discrete dynamical systems. Phys. Lett. A 262 (1999) 44-49 and: chao-dyn/9806026 .

[23] N. Abarenkova, J-C. Anglès d'Auriac, S. Boukraa and J-M. Maillard, Growth-complexity spectrum of some discrete dynamical systems. Physica D 130 (1999), 27-42 and: chaodyn/9807031. 